\title{
Análise temporal da incidência da tuberculose na população privada de liberdade
}

Temporal analysis of the incidence of tuberculosis in the private population of freedom Análisis temporal de la incidencia de tuberculosis en la población de libertad privada

Kelle Karolina Ariane Ferreira ALVES ${ }^{1}$ Lívia Menezes BORRALHO ${ }^{2}$

Ítalo de Macedo BERNARDINO

Tânia Maria Ribeiro Monteiro de FIGUEIREDO ${ }^{4}$

${ }^{I}$ Mestre em Saúde Pública pelo Programa de Pós-graduação em Saúde Pública da Universidade Estadual da Paraíba (UEPB) Campina Grande - PB, Brasil Mestre em Saúde Pública, Secretaria de Estado da Saúde, João Pessoa - PB, Brasil

${ }^{3}$ Doutorando em Odontologia, Universidade Estadual da Paraíba (UEPB) Campina Grande - PB, Brasi

${ }^{4}$ Doutora em Saúde Pública, Universidade Estadual da Paraíba (UEPB) Campina Grande - PB, Brasil

\section{Resumo}

Objetivo: verificar o comportamento da incidência da tuberculose na população privada de liberdade e estimando sua tendência. Materiais e métodos: Trata-se de um estudo ecológico de série temporal com análise de tendência da incidência da tuberculose na população privada de liberdade. Utilizou-se de dados secundários provenientes do Sistema de Informações e Agravos de Notificação. A população foi composta por todas as notificações de Tuberculose da população privada de liberdade de unidades masculinas e femininas no período de 2007 a 2016. $\mathrm{Na}$ análise de tendência temporal foi realizada através da criação de modelos de regressão polinomial e testados os modelos linear; quadrático; exponencial. Resultados: A tendência da incidência na população privada de liberdade geral e no sexo masculino foi considerada estável, ambas com $(\mathrm{p}=0,180)$, e no sexo feminino decrescente $(\mathrm{p}=0,040)$. Conclusão: É necessário avanços na condução do controle da tuberculose nas unidades prisionais.

Descritores: Tuberculose; Epidemiologia; Prisioneiros; Incidência; Saúde Pública.

\section{Abstract}

Aim: to verify the behavior of the incidence of tuberculosis in the population deprived of freedom and estimating its tendency. Materials and Methods: This is an ecological time-series study with trend analysis of the incidence of tuberculosis in the population deprived of liberty. Secondary data from the Information System on Diseases of Compulsory Declaration (Sinan) were used. The population was composed of all reports of Tuberculosis of the population deprived of freedom of male and female units in the period from 2007 to 2016 . In the analysis of temporal trend was performed through the creation of polynomial regression models and tested the linear models; quadratic; exponential. Results: The trend of the incidence in the general population of the general population and in the male population was considered stable, both with $(\mathrm{p}=0.180)$ and in the descending female sex $(\mathrm{p}=0.040)$. Conclusion: Progress is needed in the management of tuberculosis control.

Descriptors: Tuberculosis; Epidemiology; Prisoners; Incidence; Public Health.

\section{Resumen}

Objetivo: verificar el comportamiento de la incidencia de tuberculosis en la población privada de libertad y estimar su tendencia. Materiales y métodos: Este es un estudio ecológico de series de tiempo con un análisis de tendencias de la incidencia de tuberculosis en la población desfavorecida. Se utilizaron datos secundarios del Sistema de Información y Trastornos de Notificación. La población estaba compuesta por todas las notificaciones de tuberculosis de la población privada de libertad de las unidades masculinas y femeninas de 2007 a 2016. El análisis de tendencia temporal se realizó mediante la creación de modelos de regresión polinómica y los modelos lineales probados; cuadrático exponencial Resultados: La tendencia de incidencia en la población privada de libertad general y en los hombres se consideró estable, tanto con $(\mathrm{p}=0.180)$ como en mujeres en disminución $(\mathrm{p}=0.040)$. Conclusión: se necesitan avances en la realización del control de la tuberculosis en las cárceles.

Descriptores: Tuberculosis; Epidemiología; Prisioneros; Incidencia; Salud Pública.

INTRODUÇÃO

A tuberculose (TB) configura-se ainda como grave problema de saúde pública no Brasil e no mundo. Os maiores coeficientes de incidência da doença encontram-se nas populações vulneráveis, isto é: as pessoas com o Vírus da Imunodeficiência Humana (HIV), os indígenas, pessoas em situação de rua e a População Privada de Liberdade (PPL) ${ }^{1,4}$.A PPL destaca-se nesse cenário de vulnerabilidade para o adoecimento por TB pelas condições de saúde, sociais e ambientais em que são submetidas, como de baixa iluminação, ventilação e superlotação.

Estudos realizados na República Democrática do Congo e na Uganda retratam que a superlotação é frequente nas unidades prisionais de ambos os países e um dos principais fatores para o surto da doença nas prisões ${ }^{3,4}$. No Brasil,segundo dados publicados em 2018, o risco de ser acometido pela doença é 28 vezes maior na PPL do que na população geral ${ }^{5}$. O risco aumentado para se contrair a doença nas unidades prisionais pode ser explicado pelas condições do ambiente, consideradas por muitos autores como um ambiente propício na manutenção da cadeia de transmissão da doença ${ }^{6,8}$.
Atrelado às condições que favorecem a alta incidência da TB, a PPL possui um sistema de saúde prisional recente, considerado por alguns autores como subfinanciado, subequipado, com profissionais desmotivados e despreparados para atuarem no local $^{9,10}$. O que pode influenciar na busca ativa, diagnóstico precoce e tratamento da TB.

Na díade PPL-TB, em 2014, essa população foi considerada prioritária na prevenção e cuidado da TB com a aprovação da Estratégia Global da Tuberculose Pós-2015, a qual estabeleceu estratégias e metas para a redução do coeficiente de incidência em $90 \%$ e de mortalidade $95 \%$ até $2035^{11}$. Nesse sentido, torna-se necessário uma avaliação continua dos indicadores da TB na PPL, para que se possa avaliar a efetividade de políticas e estratégias já existentes e acompanhar o alcance das metas fixadas, como as da Estratégia Global Pós-2015.

No auxílio ao acompanhamento dos indicadores os gestores, profissionais e população têm a sua disposição os relatórios gerados pelo sistema de informação em saúde. No que se refere à TB há uma ampla utilização do Sistema de 
Informação de Agravos de Notificação (Sinan), que comumente é fonte para importantes estudos no âmbito nacional ${ }^{7,12,13}$.

O Ministério da Saúde, em uma de suas publicações, enfatiza que o SINAN dispõe de rotinas no intuito de qualificar seus dados no mínimo três vezes ao ano com remoção de duplicidades e vinculação de registros, executadas nas esferas estaduais e municipais para gerar o que se pode chamar de uma base de dados "qualificada". Desta forma, tornou-se uma fonte de dados importante para subsidiar o acompanhamento e investigação do comportamento da TB na população.

Diante do exposto, é oportuna e necessária a avaliação do comportamento da TB na população privada de liberdade, por meio de estudos que descrevam sua situação epidemiológica, abordando um indicador importante como a incidência. Diante da problemática, o presente estudo teve por objetivo verificar o comportamento da incidência da tuberculose na população privada de liberdade e estimando sua tendência.

\section{MATERIAL E MÉTODO}

Trata-se de um estudo ecológico de série temporal com análise de tendência da incidência da tuberculose na população privada de liberdade, aprovado por um Comitê de Ética e Pesquisa de uma universidade pública, respeitando preceitos éticos estabelecidos na Resolução 466/12, obtendo o parecer: 52879215.4.0000.5187. Utilizou-se de dados secundários provenientes do Sistema de Informações e Agravos de Notificação (SINAN). O estudo foi realizado em unidades prisionais de um estado do Nordeste brasileiro, que conta atualmente com 79 unidades prisionais, 59 cadeias públicas e 20 presídios. A população foi composta por todas as notificações de TB da PPL de unidades masculinas e femininas no período de 2007 a 2016, totalizando assim 962 notificações. Para a composição da amostra foram estabelecidos os seguintes critérios: idade maior ou igual a 18 anos e terem sido notificados como "caso novo", aplicando-se esses critérios 36 notificações foram excluídas por terem idade inferior a 18 anos e 186 por não serem casos novos. Totalizando assim uma amostra de 740 notificações. Foram considerados "casos novos" todos os doentes que não haviam se submetidos ao tratamento de TB, ou se submeteu por um período inferior a 30 dias.

No intuito de caracterizar os sujeitos do estudo realizou-se a análise estatística descritiva, com os cálculos das frequências absolutas e percentuais das características socioeconômicas, forma da doença e situação de encerramento. Para as análises de tendência foram calculadas inicialmente as incidências por ano no período estudado da população geral (número de casos novos/ PPL total do ano x 1.000), e por sexo feminino (número de casos novos na PPL no sexo feminino no ano/ PPL feminina do ano x 1.0000) e sexo masculino (número de casos novos na PPL do sexo masculino no ano/ PPL masculina do ano $x$ 1.0000), ambas as populações foram extraídas do relatório do Departamento Penitenciário Nacional (DEPEN).

Posteriormente, a análise de tendência temporal $^{14,15}$ foi utilizada para avaliar os dados, através da criação de modelos de regressão polinomial, cujo objetivo principal é encontrar a curva que melhor se adequa aos dados e descreve a relação entre a incidência de tuberculose (variável dependente) e o tempo (variável independente), o que possibilitou identificar se há tendência estável, crescente ou decrescente durante o período de observação. Os seguintes modelos de regressão polinomial foram testados: a) linear; b) quadrático; c) exponencial. A escolha do melhor modelo foi determinada pela análise do gráfico de dispersão com base no coeficiente de determinação (R2), para o qual valores mais próximos de 1 indicam melhor qualidade de ajuste, e análise de resíduos (suposição de homoscedasticidade verdadeira). Quando dois modelos foram semelhantes do ponto de vista estatístico, o modelo mais simples (ordem inferior) foi escolhido para evitar uma correlação serial entre períodos.14 O software IBM SPSS Statistics (SPSS for Windows, Version 20.0. Armonk, NY: IBM Corp.) foi utilizado para análise de dados, considerando um intervalo de confiança de $95 \%$.

\section{RESULTADOS}

A PPL do estudo era formada predominantemente por homens pardos, faixa etária de 18 a 35 anos e com 1 a 4 anos de estudo. A forma clínica de TB mais comum no período foi TB pulmonar, seguida de extrapulmonar. O encerramento mais prevalente foi cura durante todo o período estudado; o ano de 2015 se destacou com 84\%. Os óbitos por TB se destacaram em 2008, com três óbitos, correspondendo a 3,4\% (Tabelas 1 e 2).

Tabela 1. Distribuicão das características sociais e clínicas dos casos de tuberculose na população privada de liberdade, Brasil 2007-2016.

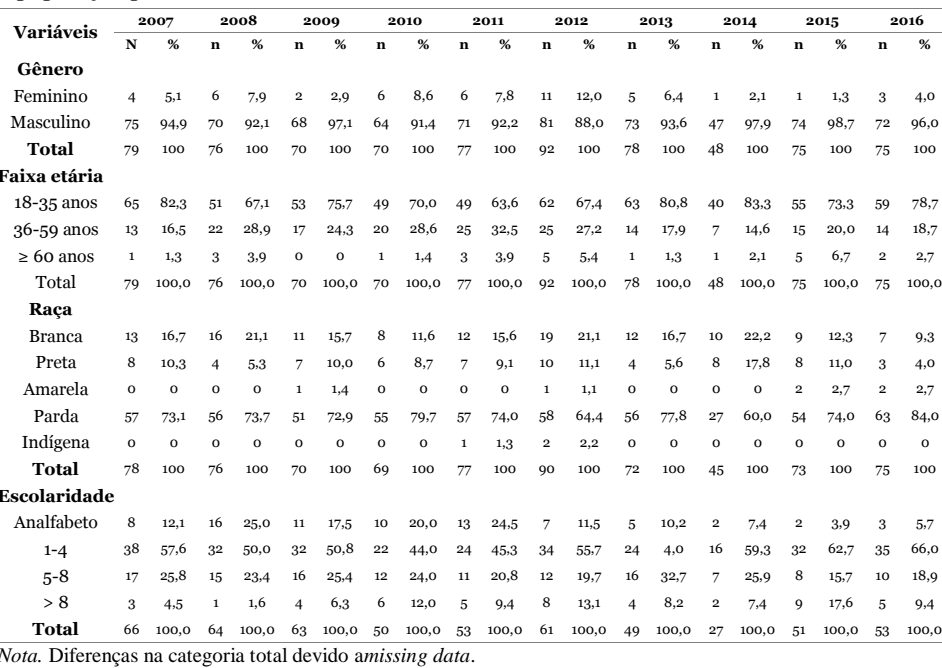

Nota. Diferenças na categoria total devido amissing data. 
Tabela 2. Distribuição das características sociais e clínicas dos casos de tuberculose na população privada de liberdade, Brasil 2007-2016.

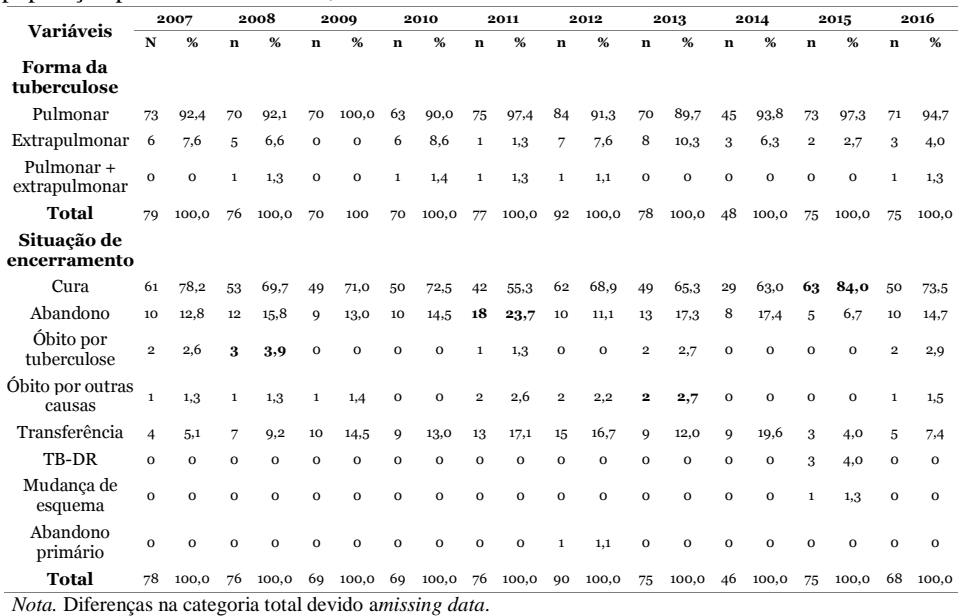

A incidência da TB no estudo foi calculada na PPL geral e por sexo. A Tabela 3 mostra a variação entre os anos estudados. Os anos que se destacaram com as maiores incidências da doença foram 2012, apresentando 11,06 na população geral, 9,94 no sexo masculino e 19,16 no sexo feminino. Os anos que se destacaram positivamente com as menores incidências na PPL geral e PPL do sexo masculino foi 2014, com 4,59 e 4,73 respectivamente; já na PPL do sexo feminino, o ano de 2015 destacou-se com 1,86, a menor incidência da série.

Tabela 3. Distribuição do coeficiente de incidência de tuberculose na população privada de liberdade (por 1000 indivíduos). Brasil, 2007-2016

\begin{tabular}{cccc} 
ANO & $\begin{array}{c}\text { Incidência anual } \\
\text { (Geral) }\end{array}$ & $\begin{array}{c}\text { Incidência anual } \\
\text { (Homens) }\end{array}$ & $\begin{array}{c}\text { Incidência anual } \\
\text { (Mulheres) }\end{array}$ \\
\hline 2007 & 9,50 & 9,32 & 14,76 \\
2008 & 8,52 & 8,14 & 18,69 \\
2009 & 8,21 & 8,36 & 5,08 \\
2010 & 8,69 & 8,43 & 13,07 \\
2011 & 9,38 & 9,31 & 10,22 \\
2012 & 11,06 & 9,94 & 19,16 \\
2013 & 8,13 & 8,22 & 6,97 \\
2014 & 4,59 & 4,73 & 1,92 \\
2015 & 7,12 & 7,40 & 1,86 \\
2016 & 6,62 & 6,72 & 4,88 \\
\end{tabular}

A Figura 1 permite uma melhor visualização do comportamento da incidência da TB por sexo na série histórica. Percebe-se que a incidência entre as mulheres a partir do ano de 2013 apresenta um declínio, o que não pode ser visto no sexo masculino.

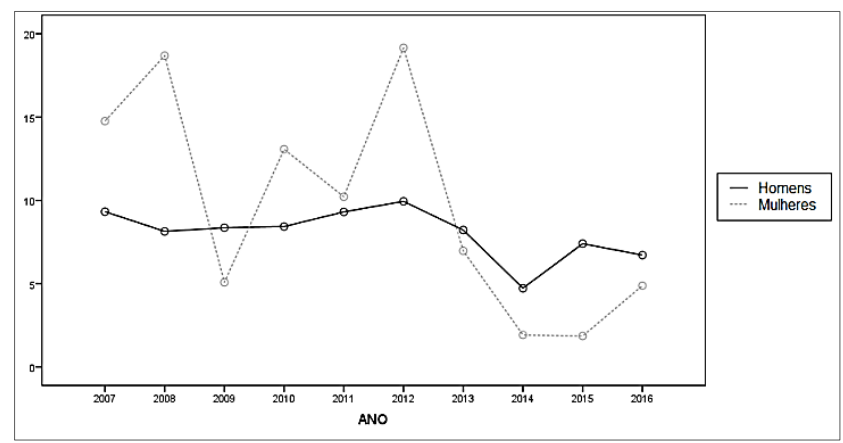

Figura 1: Distribuição do coeficiente de incidência da tuberculose da população privada de liberdade por sexo (por 1000 indivíduos). Brasil, 2007-2016
A análise de tendência da incidência de TB foi realizada na PPL geral e por sexo. Pode-se observar na Tabela 4 que a tendência na PPL geral e no sexo masculino foi considerada estável, quando ajustado o modelo de regressão quadrática, ambas as tendências foram consideradas estáveis $(\mathrm{p}=1,180)$; no sexo feminino mostrou uma tendência decrescente $(\mathrm{p}=0,040)$.

Tabela 4. Análise de tendência temporal para a incidência da tuberculose. Brasil, 2007-2016.

\begin{tabular}{|c|c|c|c|c|c|}
\hline Variável & $\mathbf{n}$ & Modelo & $\mathbf{R}^{2}$ & p-valor & Tendência \\
\hline Total & 740 & $\begin{array}{c}\mathrm{Y}=8,558+ \\
0,383 \mathrm{X}- \\
0,064 \mathrm{X}^{2}\end{array}$ & 0,388 & 0,180 & Estável \\
\hline \multicolumn{6}{|l|}{ Sexo } \\
\hline Masculino & 695 & $\begin{array}{c}\mathrm{Y}=8,555+ \\
0,247 \mathrm{X}- \\
0,048 \mathrm{X}^{2}\end{array}$ & 0,387 & 0,180 & Estável \\
\hline Feminino & 45 & $\begin{array}{c}Y=17,391- \\
1,405 \mathrm{X}\end{array}$ & 0,427 & $0,040^{*}$ & Decrescente \\
\hline
\end{tabular}

Nota. ${ }^{(a)} \mathrm{Y}=$ variável dependente (incidência anual de tuberculose); $\mathrm{X}=$ variável independente (tempo); $\mathrm{R}^{2}=$ coeficiente de determinação.

* $\mathrm{p}<0,05$

\section{DISCUSSÃ̃O}

$\mathrm{O}$ perfil dos sujeitos do estudo se assemelham a estudos recentes sobre a temática ${ }^{7,12,16}$. Com esses achados, vislumbra-se a necessidade da vigilância e atenção aos homens adultos jovens com baixa escolaridade, privados de liberdade. É oportuno destacar que existe também o problema de cunho social, com a necessidade de ações para a reinserção desses jovens em fase economicamente ativa na sociedade, gozando de plena saúde.

De maneira geral, a forma mais comum da TB é a pulmonar, o que revela preocupação, por se tratar de um espaço de confinamento e aglomerado, que colabora para o aumento da incidência e reforça a necessidade de vigilância e busca ativa dos sintomáticos respiratórios, visto a transmissão aérea do bacilo e superlotação das prisões, o que favorece a cadeia de transmissão da doença.

Estudos realizados nos Estados Unidos da América e em Moçambique destacam que a busca por casos novos na PPL trazem inúmeros benefícios a esta população, sendo o principal deles o diagnóstico precoce, que pode favorecer tanto na redução de casos novos como na adesão e cura dos doentes ${ }^{17,18}$.

Nesse mesmo pressuposto, seria esperado que especialmente nos anos de 2014 e 2015 apresentaram os maiores coeficientes de incidência, pois no ano de 2014 foi estabelecido a Política Nacional de Atenção Integral à Saúde das Pessoas Privadas de Liberdade no Sistema Prisional (PNAISP), que regulamentou o funcionamento das equipes de saúde do sistema prisional, a implantação e regulamentação das equipes viabilizariam a busca ativa, diagnósticos e notificação dos doentes, consequentemente aumentariam os coeficientes de incidência e cura o que não pode ser visto nas análises.

A Organização Mundial de Saúde estabelece como meta que as taxas de cura de TB da população 
sejam superiores a $85 \%^{19}$; essa meta não foi alcançada durante a série.

Quando analisado as tendências da incidência da TB nas populações, destaca-se a tendência decrescente na PPL feminina. Historicamente, a mulher procura com mais frequência os serviços de saúde no intuito de prevenir agravos e tratá-los. Como abordado em estudo recente que reflete que as diferenças entre os indicadores de saúde da TB entre os sexos podem ser reflexos das diferenças de comportamento e de procura aos serviços de saúde ${ }^{20}$.

As mulheres expressam maior preocupação com sua saúde e deliberam um maior tempo no cuidado, desta forma, demonstram maior adesão ao tratamento da TB. Outro ponto a ser destacado por um estudo é que a mulher tem um olhar mais voltado ao coletivo, principalmente no respeito à atenção a sua rede familiar; nesse sentido, se comprometem com o tratamento no intuito de evitar também a contaminação dos seus contatos ${ }^{21}$.

Sabe-se que no ambiente de confinamento a preocupação com os contatos também pode existir, a convivência das mulheres em um ambiente em comum pode facilitar o estreitamento de laços, fazendo com que assim, a mulher se preocupe com os contatos, além de que ainda existe o contato com seus familiares, principalmente filhos.

$\mathrm{O}$ que se faz pensar que a procura por atendimento de saúde e preocupação com seu estado de saúde pode existir também no ambiente prisional, o que vem a ser um facilitador do diagnóstico precoce no local, visto o baixo quantitativo de profissionais existentes e demora no atendimento de emergência dos privados de liberdade, problemática também destacada por um estudo recente ${ }^{22}$.

Além do comportamento social da mulher, para se explicar a estagnação na incidência da PPL masculina e geral é preciso levar em consideração que a PPL masculina numericamente é superior a PPL feminina em todo país, assim como as taxas de ocupação no local $^{23}$; um fator importante, pois se sabe que as altas taxas de ocupação propiciam uma maior transmissão da TB e dificulta as ações de saúde que visam o controle da TB. Para explicar uma possível estagnação de indicador epidemiológico em uma população especifica, é necessário se entender a fundo o contexto histórico e social da mesma e não apenas atrelar esses achados à falta de políticas públicas.

É necessário discutir as condições ambientais e estruturais em que a PPL encontra-se inserida. Alguns autores alertam que as condições sanitárias do sistema prisional brasileiro são precárias e afetam a saúde dos privados de liberdade e dos profissionais que ali atuam ${ }^{24,25}$. Além disso, a superlotação e a dinâmica funcional diferenciada do local que prioriza a segurança dos privados de liberdade, profissionais e comunidade podem dificultar a realização de ações preconizadas $^{8,26}$. Uma problemática também encontrada e discutida no cenário internacional, um estudo realizado na República da Macedônia afirma que a alta incidência de TB no ambiente prisional está diretamente relacionada às condições que favorecem a transmissão da doença ${ }^{27}$.

A PPL é constituída quase em sua totalidade de indivíduos que anteriormente viviam em áreas com pouco acesso aos serviços de saúde e com deficiências estruturais e sociais $^{28}$; todas essas questões precisam ser levadas em consideração quando se pretende avaliar os indicadores epidemiológicos. A saúde pública, com toda sua complexidade, não se limita apenas a estudar um indicador isolado; é necessário refletir todo um contexto por trás.

Em estudo realizado em 2017, a autora questiona se os esforços globais, o estabelecimento de metas quantitativas são suficientes para o combate da doença, reforça ainda a necessidade da avaliação constante do contexto histórico e social que o individuo está inserido ${ }^{29}$.

Nessa perspectiva, entende-se que o controle da TB na PLL precisa ser conduzido por políticas públicas de saúde especificas para as populações vulneráveis, pautando-se sob o princípio de equidade, compreendendo as particularidades do sistema prisional que é marcado por insalubridade e superlotação.

Este estudo trouxe importantes contribuições, pois evidenciou a necessidade do direcionamento de ações de vigilância da TB na PPL masculina, visando uma redução na incidência da doença, destacando a necessidade de ações integradas da gestão, profissionais de saúde e justiça que permitam o controle da tuberculose dentro e fora das prisões. E tem como principal limitação a subnotificação e pela incompletude dos dados no sistema de informação.

CONCLUSÃO

Observou-se uma tendência decrescente na incidência da TB na PLL do sexo feminino e estável na PLL do sexo masculino e geral, desta forma percebe-se que é necessário avanços na condução do controle da TB nas unidades prisionais, principalmente masculinas; esse avanço só será possível mediante mudanças não apenas com políticas públicas específicas para a população que compreendam suas particularidades estruturais e funcionais, mas principalmente na melhoria das condições de insalubridade em que os privados de liberdade estão expostos superlotações e fragilidades das equipes de saúdes prisionais.

\section{REFERÊNCIAS}

1. Brasil. Ministério da Saúde. Secretaria de Vigilância em Saúde. Departamento de Vigilância Epidemiológica. Manual de recomendação para o controle da tuberculose no Brasil. Brasília: 
Ministério da Saúde. 2018.

2. World Heatlh Organization. 2017 Fer.Disponívelem: http://www.who.int/tb/areasof-work/population-groups/prisons-facts/en/.

Acesso em : 20 Jan. 2017.

3. Kayomo MK, Hasker E, Aloni M, Nkuku L, Kazadi M, Kabengele $T$, et al. Outbreak of Tuberculosis and Multidrug-Resistant Tuberculosis, Mbuji-Mayi Central Prison, Democratic Republic of the Congo. Emerg Infect Dis. 2018;24(11):2029-35.

4. Schwitters A, Kaggwa M, Omiel P, Nagadya G, Kisa N, Dalal S. Tuberculosis incidence and treatment completion among Ugandan prison inmates. Int J Tuberc Lung Dis. 2014;18(7):781-86.

5. Brasil. Ministério da Saúde. Secretaria de Vigilância em Saúde. Boletim epidemiológico. Brasília: Ministério da Saúde, 2018;49(8).

6. Alinaghi SAS, Farhoudi B, Najafi Z, Jafari S. Comparing Tuberculosis incidence in a prison with the society, Tehran, Iran. Arch Clin Infect Dis. 2018;E60247:1-3.

7. Sacramento DS, Gonçalves MJF. Situação da tuberculose em pessoas privadas de liberdade no período de 2007 a 2012 . J Nurs UFPE on line. 2017;11(1):140-51.

8. Valença MS, Possuelo LG, Cezar-Vaz MR, Silva PE. Tuberculose em presídios brasileiros: uma revisão integrativa da literatura. Cien Saude Colet. 2016;21(7):2147-60.

9. Sánchez A, Larouzé B. Tuberculosis control in prisons, from research to action: the Rio de Janeiro, Brazil, experience. Cien Saude Colet. 2016;21(7):2071-80.

10. Martins ELC, Martins LG, Silveira AM, Melo EM. The contradictory right to health of people deprived of liberty: the case of a prison in Minas Gerais , Brazil. Saúde soc. 2014;23(4):1222-34.

11. Ministério da Saúde (BR). Secretaria de Vigilância em Saúde. Departamento de Vigilância das Doenças Transmissíveis. Brasil livre da tuberculose. Plano nacional pelo fim da tuberculose como problema de saúde pública [Internet]. Brasília: Ministério da Saúde; 2017 [citado 2018 mar 8]. 52 p. Disponível em: https://drive.google.com/file/d/0B0CE2wqdEaReVc5V3cyMVFPcTA/view.

12. Macedo LR, Maciel ELN, Struchiner CJ. Tuberculose na população privada de liberdade do Brasil, 2007-2013*. Epidemiol Serv Saúde. 2017;26(4):783-94.

13. Silva PF, Moura GS, Caldas AJM. Fatores associados ao abandono do tratamento da tuberculose pulmonar no Maranhão, Brasil, no período de 2001 a 2010. Cad Saúde Pública. 2014;30(8):1745-54.

14. Montgomery DC, Jennings CL, Kulahci M. Introductionto Time Series Analysis and
Forecasting. 2th ed. Hoken, NJ: John Wiley\&Sons; 2015.

15. Cavalcante GMS, de Macedo Bernardino Í, da Nóbrega LM, Ferreira RC, Ferreira E Ferreira E, d'Avila S. Temporal trends in physical violence, gender differences and spatial vulnerability of the location of victim's residences. Spat Spatiotemporal Epidemiol. 2018;25:49-56.

16. Alves JP, Brazil JM, Nery AA, Vilela ABA, Filho IEM. Perfil Epidemiológico de pessoas privadas de liberdade. Rev enferm UFPE on line. 2017;11(supl.10):4036-44.

17. Lambert LA, Armstrong LR, Lobato MN, Ho C, France AM, Haddad MB. Tuberculosis in Jails and Prisons: United States. AJPH Res. 2016;106(12):2231-37.

18. Orlando S, Triulzi I, Ciccacci F, Palla I, Palombi L, Marazzi MC et al. Delayed diagnosis and treatment of tuberculosis in $\mathrm{HIV}+$ patients in Mozambique: A cost-effectiveness analysis of screening protocols based on four symptom screening, smear microscopy, urine LAM test and Xpert MTB/RIF. PLoS One. 2018;13(7):1-16.

19. World HeatlhOrganization. The end TB strategy [Internet]. Geneva: World HeatlhOrganization; 2015. 20 p. Available in: http://www.who.int/tb/End_TB_brochure.pdf

20. Belo MTCT, Luiz RR, Hanson SL, Teixeira EG, Chalfoun T, Trajman A. Tuberculose e gênero em um município prioritário no estado do Rio de Janeiro. J Bras Pneumol. 2010;36(5):621-25.

21. Sá LD, Santos ARBN, Oliveira AAV, Nogueira JA, Tavares LM, Villa TCS. O cuidado á saúde da mulher com tuberculose na perspectiva do enfoque familiar. Texto contexto - enferm. 2012;21(2):409-17.

22. Minayo MCS, Ribeiro AP. Condições de saúde dos presos do estado do Rio de Janeiro, Brasil Health conditions of prisoners in the state of Rio de Janeiro , Brazil. Ciênc saúde coletiva. 2016;21(7):2031-40.

23. Brasil. Ministério da Justiça e Segurança Pública. Departamento Penitenciário Nacional. Levantamento Nacional de Informações Penitenciárias: INFOPEN atualização junho de 2016. Org. Tandhara Santos; Colaboração. Marlene Inês da Rosa, et al. Brasília - DF, 2017, p. 65

24. Winter BCA, Grazinoli Garrido R. A tuberculose no cárcere: um retrato das mazelas do sistema prisional brasileiro. Med leg Costa Rica. 2017;34(2):20-31.

25. Soares Filho MM, Bueno PMMG. Demography, vulnerabilities and right to health to Brazilian prison population. Cien Saude Colet. 2016;21(7):1999-2010.

26. Santos MNA, Sá AMM. Viver com tuberculose em prisões: $\mathrm{O}$ desafio de curar-se. Texto contexto 
- enferm. 2014;23(4):854-61.

27. Ilievska-Poposka B, Zakoska M, PilovskaSpasovska K, Simonovska L, Mitreski V. Tuberculosis in the Prisons in the Republic of Macedonia, 2008-2017. Maced J Med Sci. 2018;6(7):1300-4.

28. Oliveira LGD, Natal S, Camacho LAB. Contextos de implantação do Programa de Controle da Tuberculose nas prisões brasileiras. Rev Saúde Pública. 2015;49:66.

29. da Silva RD, de Luna FDT, de Araújo AJ, Camêlo ELS, Bertolozzi MR, Hino P, Lacerda SNB, Fook SML, de Figueiredo TMRM. Patients' perception regarding the influence of individual and social vulnerabilities on the adherence to tuberculosis treatment: a qualitative study. BMC Public Health. 2017;17(1):725.

\section{CONFLITO DE INTERESSES}

Os autores declaram não haver conflitos de interesse.

\section{AUTOR PARA CORRESPONDÊNCIA}

Kelle Karolina Ariane Ferreira Alves

Rua Eliezer Dourado n ${ }^{\circ} 355$, Coopirecê 49900-000 Irecê- Bahia, Brasil

kellekarolina@hotmail.com

Submetido em 20/01/2020

Aceito em 15/12/2020 